\title{
Prévention du cancer colorectal : le changement c'est (vraiment) maintenant !
}

\author{
Prevention of the colorectal cancer: change is right now!
}

\author{
G. Lesur \\ C Springer-Verlag France 2014
}

Que ceux et celles qui craignent en lisant ce titre un éditorial politique soit rassuré, vous pouvez continuer votre lecture. De politique au sens traditionnel il ne sera pas question, mais de la gestion des affaires publiques, ce qui est la définition noble de la politique, il sera bien question. Car oui, cela devrait enfin bouger dans la prévention du cancer colorectal en 2014. Le changement, c'est vraiment pour maintenant!

Les chiffres catastrophiques du cancer colorectal en France sont connus de tous et avec 40000 nouveaux cas par an, dont 17000 vont mourir chaque année, il s'ag it d'une véritable hécatombe. Il faut absolument faire chuter drastiquement ces chiffres. Des données scientifiques récemment publiées nous éclairent à nouveau sur les moyens d'y arriver.

Le premier travail confirme l'efficacité à très long terme du dépistage par test Hemoccult ${ }^{\circledR}$, en montrant que la réalisation d'un test annuel ou biennal permet la réduction de la mortalité de respectivement $32 \%$ et $22 \%$ par rapport à un groupe de sujets non dépistés [1]. Ce résultat a été obtenu avec le vieux test Hemoccult ${ }^{\circledR}$ dont on connait le manque de sensibilité et de spécificité. De très nombreux pays ont déjà fait le choix de remplacer ces tests anciens par les tests immunologiques aux performances meilleures. Malgré l'avis favorable rendu sur ces tests immunologiques par la HAS en 2009 et une prévision de passage à ces tests en 2013, rien ne s'est malheureusement passé en France. Mais une première avancée vient d'être obtenue car des appels d'offres pour la mise en place des tests immunologiques, préambule indispensable avant leur diffusion, devraient enfin être lancés. On espère que cette première étape sera rapide et surtout qu'elle sera suivie d'une diffusion accélérée de ces tests plus performants. Mais il va falloir maintenir la pression jusqu'à l'amélioration vraie de ce premier volet de la

G. Lesur $(\bowtie)$

Hôpital Ambroise Paré, 9, avenue Charles de Gaulle,

F-92104 Boulogne-Billancourt cedex

e-mail : gilles.lesur@apr.aphp.fr prévention du cancer colorectal. Toutefois, la disponibilité de tests meilleurs ne résoudra pas tout dans un pays où le taux d'adhésion aux tests actuels, dont il est vrai que les insuffisances sont maintenant connues, est d'environ $30 \%$. Il y aura donc de nouveau un important travail d'information à faire auprès de nos concitoyens comme de nos collègues généralistes.

Mais comme tout bon responsable de famille, ne mettons pas tous nos œufs dans le même panier. Car un autre papier récent démontre que la coloscopie et la rectosigmoïdoscopie réduisent de respectivement $70 \%$ et $40 \%$ le risque d'avoir un cancer colorectal [2]. De plus, cette réduction du risque d'avoir un cancer persiste pendant dix ans chez les sujets sans antécédent familial et pendant cinq ans en présence d'un antécédent familial de cancer colorectal. Ce travail démontre aussi que la coloscopie totale est seule susceptible de réduire le risque de cancer du côlon proximal, même si cette réduction est moins importante puisque d'environ $20 \%$.

$\mathrm{Au}$ moment où vous lirez cet éditorial, le nouveau « Plan Cancer » aura été dévoilé publiquement. Espérons qu'y figurent enfin ces tests immunologiques et pourquoi pas, il n'est pas interdit de rêver, une place pour la coloscopie qui fait naturellement partie de l'arsenal de la lutte contre le cancer colorectal. Deux méthodes aux finalités et à la mise en œuvre différentes, en symbiose ou non, sont nécessairement plus fortes qu'une approche unique pour espérer faire chuter l'incidence d'une maladie aussi fréquente et grave, et aisément accessible à la prévention que le cancer colorectal.

Quant à nous, gastroentérologues, il nous faudra aussi chaque jour exiger et obtenir des préparations coliques excellentes, prendre son temps pour examiner le mieux possible le côlon, progresser dans la reconnaissance de ces redoutables polypes du côlon droit qui peuvent être sources de cancers d'intervalle, faire mieux dans les résections endoscopiques de polypes (des résections incomplètes étant elles aussi causes de cancers d'intervalle), évaluer nos taux de détection des adénomes, utiliser des endoscopes haut de gamme et les techniques de coloration pour à la fois dépister, 
reconnaître et réséquer puis surveiller. Ce n'est qu'au prix de cette mobilisation de tous les instants, et qui nous concerne tous que, tous ensemble, nous réussirons ce combat contre le cancer colorectal.

Au nom du conseil d'administration de la SFED et du comité de rédaction d'Acta Endoscopica, je vous souhaite une bonne et heureuse année 2014.

\section{Références}

1. Shaukat A, Mongin SJ, Geisse MS, Lederle FA, Bond JH, Mandel JS, et al. Long-term mortality after screening for colorectal cancer. N Engl J Med 2013;369:1106-14.

2. Nishihara R, Wu K, Lochhead P, Morikawa T, Liao X, Qian ZR, et al. Long-term colorectal cancer incidence and mortality after lower endoscopy. N Engl J Med 2013;369:1095-105.

Vous venez de soutenir votre mémoire de DES ?

Votre mémoire est consacré à l'endoscopie digestive ?

Publiez-le en français dans Acta Endoscopica

Soumettez vos manuscrits sur : https:/www.editorialmanager.com/acen/ 